\title{
SUR UN SPOROTRICHUM AGENT DE LA MATURATION NORMALE DE CERTAINS FROMAGES (1)
}

par

\author{
G. GUITTONNEAU \\ Suzanne HAAS \\ Directeur \\ Chef de travaux \\ du Laboratoire national des Industries laitières
}

\section{INTRODUCTION}

Six semaines environ après la fabrication des fromages du type Saint-Nectaire, apparaissent sur la croûte des taches jaunes soufre que les spécialistes considèrent comme un indice de qualité pour ces fromages. Il était normal de penser que ces taehes étaient constituées par un organisme ayant un rôle dans la maturation. Nous en avons entrepris l'isolement. Dans plus de dix cas distincts, on a toujours obtenu le même organisme, qu'accompagnaient de nombreux types de moisissures. Il s'agit, en effet, d'une moisissure s'apparentant, en première analyse, au groupe des imperfecti et dont il restait à faire l'identification.

Des ensemencements de cet organisme effectués sur un fromage de Saint-Nectaire aussitôt après sa sortie de la presse ont permis d'obtenir un fromage de qualité excellente et entièrement recouvert de taches jaunes. Ce fromage est représenté par la photographie de la figure 1.

Sur l'ensemble des souches isolées, une seule a été retenue comme type. Elle provenait d'un Saint-Nectaire de premier choix. Nous la désignerons par la lettre $\mathrm{N}$.

\section{ÉTUDE MORPHOLOGIQUE DE LA SOUCHE N}

\section{Aspect macroscopique sur divers milieux de culture}

Sur tranche de carotte ou de pomme de terre stérilisées, la souche $\mathrm{N}$ prolifère très bien et l'ensemble de la culture prend une coloration jaune vif assez persistante. Sur milieu de Sabouraud (gélose nutritive maltosée), la prolifération est très forte. Les colonies sont géantes et s'incrustent profondément dans le gel. En surface du milieu, elles forment des reliefs très accentués. Les colonies se bordent de zones de colorations différentes allant du jaune à l'orange et même au vert pâle. Sur gélose nutritive à base de lait écrémé préalablement digéré par la trypsine [1] et considéré par nous comme un milieu de

(1) Ce travail, publié également dans les Annales de Technologie agricole, 1940, 3, a fait l'objet d'une communication à l'Académie des Sciences, $C$. $R$. Académie des Sciences, 1939, CCVIII, 1343. 


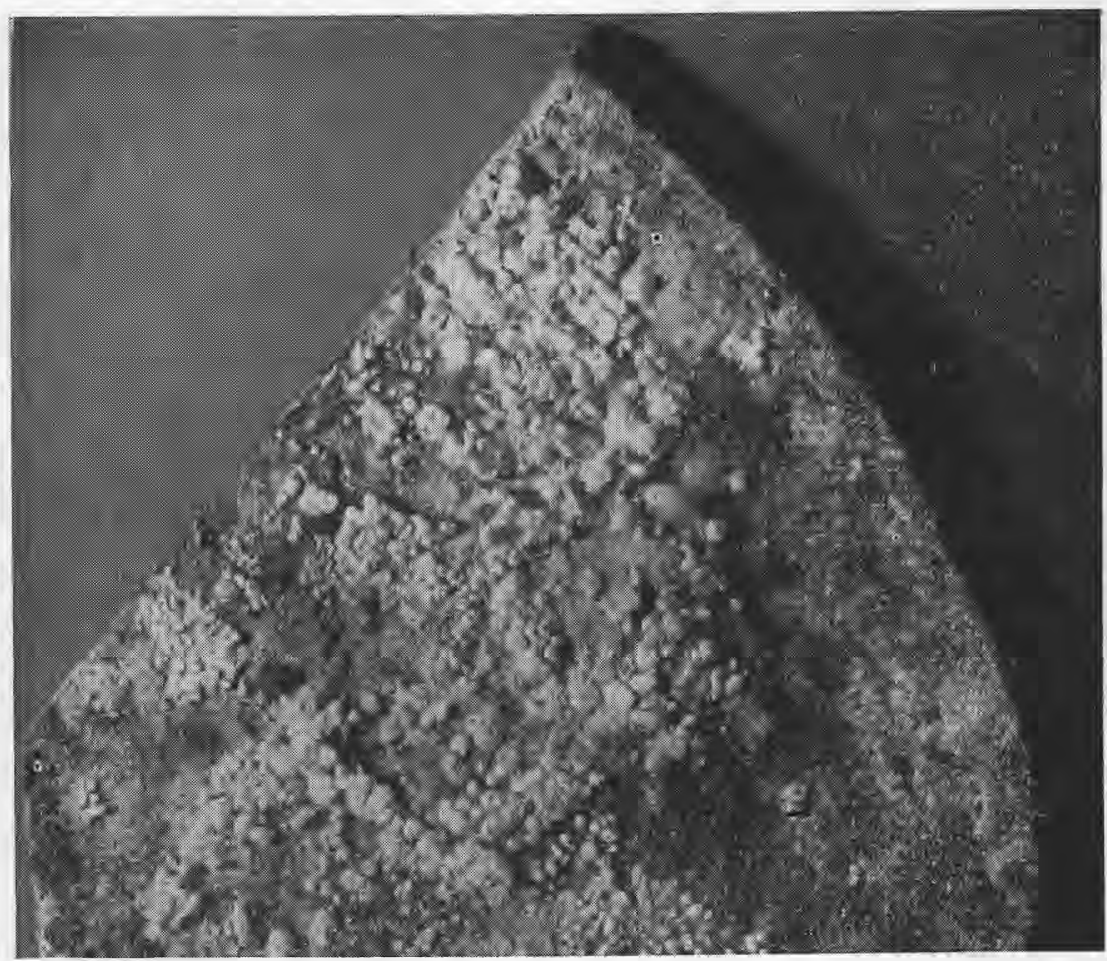

Fig. 1.

choix pour toutes les études de microbiologie fromagère, le développement se fait très bien également.

En milieu liquide tel que le lait ou le liquide de Raulin enrichi de peptone, la moisissure forme dans la masse du liquide des amas lenticulaires de filaments enchevêtrés. La sporulation ne se produit pas, et les filaments restent généralement incolores.

\section{Aspect microscopique. - Cycle évolutif}

$\mathrm{Au}$ microscope, les cultures montrent un feutrage mycélien portant des conidies à peu près sphériques et de diamètre assez régulièrement compris entre 5 et $6 \mu$.

Nous avons pu suivre, sous le microscope et à la température du laboratoire, le développement des conidies incluses dans une gouttelette de gélose nutritive à base de lait écrémé digéré par la trypsine, Elles germent, en général, après 18 heures d'incubation, donnant naissance chacune à un ou plusieurs filaments mycéliens qui commencent à se cloisonner à partir du $3^{\mathrm{e}}$ jour et forment, à partir du $5^{\mathrm{e}}$ jour, des conidies latérales munies ou non d'un court 
pédicelle. Vers le $8^{\mathrm{e}}$ jour les conidies se séparent des filaments mycéliens. Ces conidies présentent, dans les vieilles cultures, un aspect verruqueux provoqué par le rassemblement à leur périphérie de granulations pigmentaires jaunes. (L'ensemble du développement est représenté par la fig. 2.)

\section{Identification morphologique de l'espèce}

L'ensemble des caractères que l'on vient de mentionner permet de classer la souche $\mathrm{N}$ dans le genre Sporotrichum et de l'identifier à l'espèce que Link a décrite en 1809 sous le nom de Sporotrichum aureum [2]. Jusqu'ici, la présence de cette espèce avait surtout été signalée sur des débris organiques en voie d'altération. Le plus souvent, sur des substances d'origine végétale telles que le bois, les écorces, les feuilles, les champignons, et, une fois au moins, en 1937, à Baarn (Hollande), sur des tissus animaux (patte de crapaud desséchée trouvée dans une cave).

Aucune étude physiologique de cette espèce n'a été publiée. Aussi avons-nous jugé intéressant de soumettre, parallèlement, à quelques études biologiques, notre souche isolée du Saint-Nectaire (souche N) et la souche hollandaise de Baarn (souche B).

\section{ÉTUDE BIOLOGIQUE COMPARÉE DE LA MOISISSURE IDENTIFIÉE ET D'UNE SOUCHE DE RÉFÉRENCE}

\section{Observations sur les méthodes d'étude adoptées}

La moisissure étudiée pousse beaucoup plus facilement sur un support solide qu'en milieu liquide où elle forme généralement, ainsi que nous l'avons déjà dit, des petites lentilles incolores de filaments non sporifères.

Pour conserver les avantages qu'offrent les milieux liquides au point de vue des études analytiques, nous avons eu recours à l'artifice suivant : un support inerte, formé de coton de verre, soigneusement lavé à l'acide chlorhydrique et à l'eau, était introduit dans une fiole d'Erlenmeyer. Après stérilisation de la fiole et de son support, on apportait aseptiquement une quantité connue de la solution nutritive stérile à étudier. Les fioles étaient fermées au moyen d'un bouchon de caoutchoue perforé qui laissait passer un tube de verre, à boule, obturé à son extrémité supérieure par une bourre de coton. Après ensemencement, les bouchons de caoutchouc sont préservés extérieurement par du coton stérile maintenu par du papier. Ce dispositif permet de supprimer les pertes d'eau par évaporation.

\section{Alimentation hydrocarbonée des souches $\mathbf{N}$ et $\mathbf{B}$}

Des essais de culture sur liquide de Raulin et différentes autres solutions minérales ont permis de mettre en évidence l'impossibilité, 

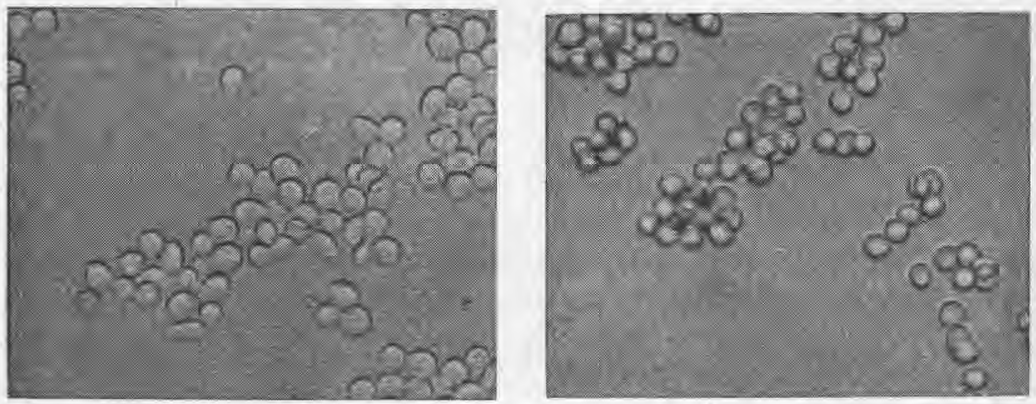

Spores.
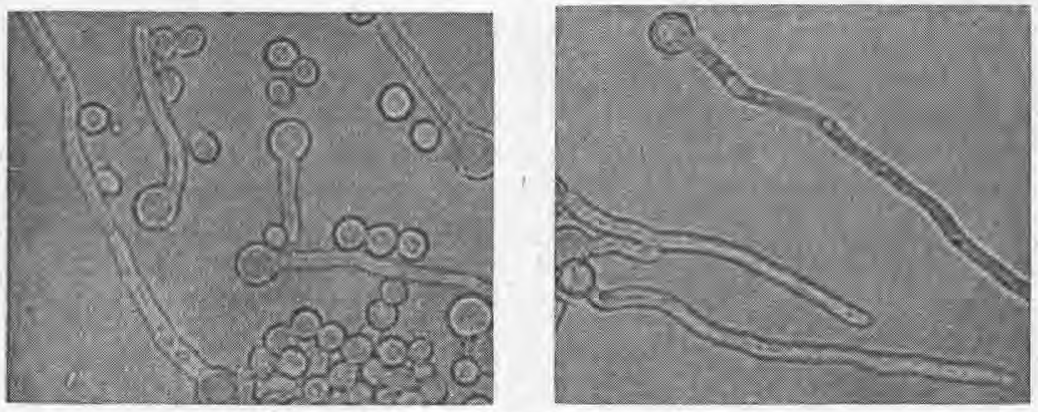

Germination des spores.
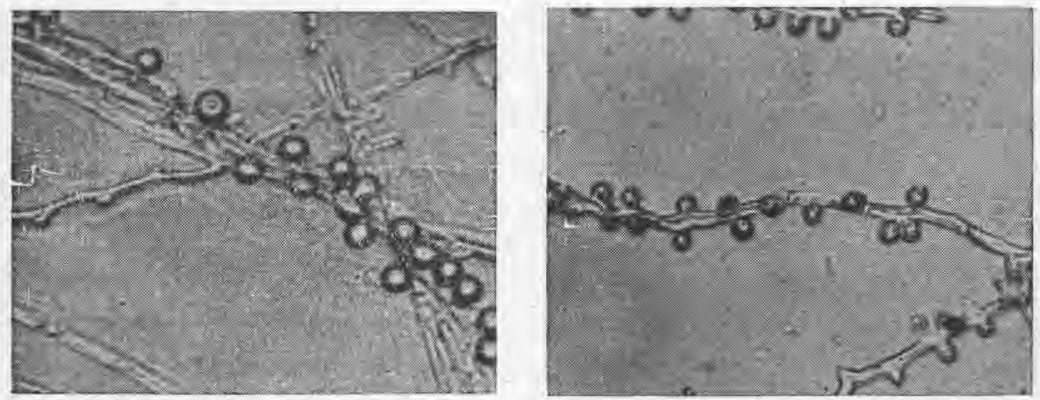

Conidies latérales.


Désagrégation des filaments.

s Souche N.

Souche B.

Fig. 2. 
où sont ces moisissures de se développer sans azote organique. Le liquide de Raulin peptoné est, par contre, un bon milieu de culture.

Les essais de nutrition hydrocarbonée n'ont donc pu être effectués qu'en présence d'azote organique.

Comme, par ailleurs, le milieu de Sabouraud assure une excellente croissance au Sporotrichum, nous ayons utilisé pour les essais la peptone granulée de Chassaing (qui sert à la préparation du milieu de Sabouraud), à laquelle nous ajoutons l'un des sucres à étudier. La peptone est apportée sous forme d'une solution à $1 \% .20 \mathrm{~cm}^{3}$ de cette solution sont versés dans une fiole décrite au paragraphe précédent. On y ajoute $20 \mathrm{~cm}^{3}$ de solution sucrée à $2 \%$ environ et stérile. On prélève aseptiquement $5 \mathrm{~cm}^{3}$ du mélange sur lesquels on dose le sucre par la méthode Bertrand afin de connaître la quantité initiale existant dans chaque fiole.

Le même mélange de solution sucrée et peptonée est introduit dans trois fioles identiques. L'une est ensemencée avec la souche $\mathrm{N}$, l'autre avec la souche $B$, et la troisième sert de témoin. On constatait généralement que la souche $B$ se développait plus rapidement que la souche $\mathrm{N}$.

Après 45 jours de culture à la température du laboratoire, on ramenait le contenu de chaque fiole à un même volume et on dosait la quantité de sucre restant.

Par différence avec la teneur en sucre du témoin on déterminait la quantité de sucre consommé (voir le tableau I).

\section{TABLEAU I}

SUGRE GONSOMMÉ \% DU SUGRE DANS LE MILIEU DE GULTURE

\begin{tabular}{|c|c|c|c|c|c|c|c|c|}
\hline & $\begin{array}{c}\text { Saccha- } \\
\text { rose }\end{array}$ & $\begin{array}{l}\text { Mal- } \\
\text { tose }\end{array}$ & $\begin{array}{l}\text { Lac- } \\
\text { tose }\end{array}$ & $\begin{array}{l}\text { Glu- } \\
\text { cose }\end{array}$ & $\begin{array}{l}\text { Fruc- } \\
\text { tose }\end{array}$ & $\begin{array}{c}\text { Galac- } \\
\text { tose }\end{array}$ & $\begin{array}{c}\text { Arabi- } \\
\text { nose }\end{array}$ & Xylose \\
\hline ouche $\mathrm{N}$ & 7,4 & 93,8 & 5,8 & 98 & 97,9 & 93,5 & 63,2 & 66 \\
\hline Souche B & 7,9 & 91,6 & 5,8 & 96 & 95,9 & 95,5 & 56,1 & 55,1 \\
\hline
\end{tabular}

On voit par ce tableau que les deux souches se comportent très sensiblement de la même manière, vis-à-vis des liquides nutritifs étudiés.

\section{Alimentation azotée des souches $\mathbf{N}$ et $\mathbf{B}$}

Les expériences entreprises ont permis de définir exactement les besoins en azote de ces organismes.

Le même dispositif expérimental que pour l'étude des sucres a été adopté.

Les milieux de culture étaient composés d'une solution minérale renfermant : 
Pour un litre :



additionnée, en partie égale, d'une solution azotée renfermant soit 18 gr. 8 de sulfate d'ammoniaque par litre, soit 28 gr. 8 de nitrate de potasse, soit $8 \mathrm{gr}$. 4 d'urée, de façon que le milieu de culture renferme, dans tous les cas, un gramme d'azote par litre.

Enfin, les souches furent ensemencées sur ces divers milieux tels quels ou additionnés de glucose.

Dans aucun cas on n'a constaté le moindre développement.

Si l'on utilise des milieux liquides aux acides aminés tels que :

0 gr. 315 d'alanine;

0 gr. 04 de sulfate de magnésie ;

0 gr. 04 de phosphate monopotassique;

50 centimètres cubes d'eau ;

ou la même solution minérale enrichie, soit de $0 \mathrm{gr}$. 46 de leucine ou de 0 gr. 47 d'acide aspartique, ou de 0 gr. 22 de tryptophane, ou de 0 gr. 16 de tyrosine, on ne constate aucune prolifération des souches $\mathrm{N}$ et $\mathrm{B}$.

$\mathrm{Si}$, au contraire, on solidifie les mêmes milieux de culture à l'aide de gélose lavée, on constate, après 20 jours de culture à $21^{\circ} \mathrm{C}$., sur tous, un développement qui, sans être luxuriant, indique l'aptitude de ces organismes à utiliser l'azote de certains acides aminés. L'alanine, la tyrosine donnent un développement très net. Ce développement est moindre avec la leucine, il est lent et faible avec le tryptophane.

Les milieux témoins sans azote n'ont jamais donné lieu à aucune végétation.

Enfin, les peptones ont été employées comme aliment azoté dans l'expérience sur l'alimentation hydrocarbonée des souches $\mathrm{N}$ et $\mathrm{B}$. Les dosages d'azote au microkjeldahl ont été effectués en présence des divers sucres mis en expérience.

On a exprimé l'azote total et l'azote ammoniacal. Pour ce dernier, on a adopté la méthode par déplacement à l'ébullition en présence de magnésie de préférence à la méthode par entraînement à froid a u moyen d'un courant d'air purifié. Les deux nous ayant donné, après quelques essais préalables, des résultats parfaitement comparables, 
nous avons retenu la première qui présente l'avantage d'être la plus rapide.

TABLEAU II

attaque de la matiěre azotée (PEPTONE), EN PRÉSence de sucre

\begin{tabular}{|c|c|c|c|c|}
\hline & & $\begin{array}{l}\text { Témoin } \\
\mathrm{N} \% \%^{\circ} \\
\text { Grammes }\end{array}$ & $\begin{array}{c}\text { Souche B } \\
\text { N\%o restant } \\
\text { Grammes }\end{array}$ & $\begin{array}{c}\text { Souche } \mathrm{N} \\
\mathrm{N} \% \text { \% restant } \\
\text { Grammes }\end{array}$ \\
\hline \multirow{2}{*}{ Glucose } & $\mathrm{N}$ total $\ldots \ldots \ldots \ldots$ & 1,360 & 0,940 & 0,960 \\
\hline & $\mathrm{N} \mathrm{H}^{3} \ldots \ldots \ldots \ldots$ & 0,032 & 0,4 & 0,4 \\
\hline \multirow{2}{*}{ Lévulose } & $\mathrm{N}$ total $\ldots \ldots$ & 1,360 & 0,820 & 0,860 \\
\hline & $\mathrm{N} \mathrm{H}^{3} \ldots$ & 0,032 & 0,304 & 0,5 \\
\hline \multirow{2}{*}{ Galactose } & $\mathrm{N}$ total .......... & 1,360 & 0,800 & 0,860 \\
\hline & $\mathrm{N} \mathrm{H}^{3}$ & 0,032 & 0,272 & 0,24 \\
\hline \multirow{2}{*}{ Saccharose } & $\mathrm{N}$ total $\ldots \ldots$ & 1,180 & 1,120 & 1,130 \\
\hline & $\mathrm{N} \mathrm{H}^{3} \ldots$ & 0,016 & 0,800 & 0,815 \\
\hline \multirow{2}{*}{ Lactose } & $\mathrm{N}$ total & 1,140 & 1,100 & 1,140 \\
\hline & $\mathrm{N} \mathrm{H}^{3}$ & $.0,040$ & 0,760 & 0,792 \\
\hline \multirow{2}{*}{ Maltose } & $\mathrm{N}$ total & 1,360 & 0,760 & 0,900 \\
\hline & $\mathrm{N} \mathrm{H}^{3}$ & 0,082 & 0,264 & 0,368 \\
\hline \multirow{2}{*}{ Xylose. } & $\mathrm{N}$ total $\ldots \ldots \ldots$ & 1,680 & 0,920 & 1,040 \\
\hline & $\mathrm{N} \mathrm{H}^{3} \ldots \ldots \ldots \ldots$ & 0,040 & 0,552 & 0,400 \\
\hline \multirow{2}{*}{ Arabinose } & $\mathbf{N}$ total & 1,680 & 1,020 & 1,000 \\
\hline & $\mathrm{N} \mathrm{H}^{3} \ldots \ldots \ldots \ldots$ & 0,040 & 0,576 & 0,528 \\
\hline
\end{tabular}

\section{Attaque des éléments du lait par les souches $\mathbf{N}$ et $\mathbf{B}$}

Le lactose étant à peine attaqué, l'activité des deux moisissures se porte surtout sur les protéides et notamment sur la caséine. Le liquide perd rapidement son opacité et s'enrichit progressivement en ammoniaque. Le tableau III donne le résultat des dosages effectués après 16 jours de culture sur lait.

Pour donner une idée de l'état de dégradation de l'azote, on a déterminé ici les trois fractions suivantes : azote total, azote non précipitable par l'acide trichloracétique et azote ammoniacal.

TABLEAU III

BILAN ALIMENTAIRE DES SOUGHES N ET B SUR LAIT

\begin{tabular}{|c|c|c|c|c|}
\hline$=$ & $\begin{array}{c}\text { Sucre } \%{ }^{\circ} \\
\text { restant. } \\
\text { Grammes }\end{array}$ & $\begin{array}{c}\text { N Total } \\
\% \%^{\circ} \\
\text { Grammes }\end{array}$ & $\begin{array}{c}\mathrm{N} \text { non } \\
\text { précipitable } \\
\text { par l'acide } \\
\text { trichloracé- } \\
\text { tique. } \\
\text { Grammes }\end{array}$ & $\begin{array}{c}\mathrm{N} \\
\text { ammoniacal. } \\
\text { Grammes }\end{array}$ \\
\hline Témoin ....... & 46,7 & 5,85 & 0,37 & 0,14 \\
\hline Souche B. . . . . & 41,3 & 5,83 & 1,75 & 0,224 \\
\hline Souche $\mathbf{N} \ldots \ldots$ & 44,8 & 5,71 & 1,42 & 0,17 \\
\hline
\end{tabular}




\title{
Comportement des souches $\mathbf{N}$ et $\mathbf{B}$ dans l'échelle des températures
}

Ensemencées sur le milieu gélosé à base de lait digéré par la trypsine, les deux souches ont été maintenues à diverses températures.

A $7^{\circ} \mathrm{C}$., on n'a noté qu'un très léger départ de végétation vers le $5^{\mathrm{e}}$ jour et la culture n'a pas évolué.

A $12^{\circ} \mathrm{C}$., le départ se produit vers le $2^{\mathrm{e}}$ jour et la culture évolue normalement, bien qu'assez lentement.

A $21^{\circ} \mathrm{C}$., la croissance est notable dès le $2^{\mathrm{e}}$ jour et progresse rapidement avec jaunissement dès le $5^{\mathrm{e}}$ jour.

A $23^{\circ} \mathrm{C}$, , le développement est encore plus rapide.

A $30-37$ et $45^{\circ} \mathrm{C}$., les moisissures ne poussent plus du tout.

La zone optima est donc comprise entre 21 et $23^{\circ} \mathrm{C}$., elle est très voisine de la température maxima $\left(30^{\circ} \mathrm{C}\right.$.).

\section{Conclusion}

La présence constante du Soprotrichum aureum dans la maturation des fromages du type Saint-Nectaire autorise à penser que cette moisissure joue un rôle important dans l'affinage de ces fromages.

Après l'identification morphologique de cet organisme, l'étude biologique que nous avions entreprise avait pour but de mettre en évidence l'existence d'une "race » de Sporotrichum aureum spécialement adaptée à la vie sur les fromages. Or, les ressemblances physiologiques que nous avons relevées entre les souches $N$ et $B$ ne nous permettent pas, pour le moment, de retenir cette manière de voir.

\section{BIBLIOGRAPHIE}

[1] G. Guittonnead, P. Sajous et P. De Peet. Recherche d'un milieu de culture approprié à la bactériologie fromagère. Le Lait, 1930, juinjuillet, p. 614 ; août-septembre, p. 750 .

[2] LiNk. Observationes in ordines plantarum naturales. Dissertatio I dans Magaz. Gesamter Naturforschung Freunde, Berlin III, 13, 1809.

\section{UN PROCÉDÉ AMERICAIN POUR LA FABRICATION DE LA LAINE DE CASÉINE}

\author{
par \\ G. GÉNIN. \\ Ingénieur E. P. C.
}

A la suite des premières recherches de Millar [1] et de TodtenHAUPT [2] sur la fabrication de la laine artificielle, en partant de caséine, on sait que c'est en Italie, grâce aux travaux de FErRetri [3], 